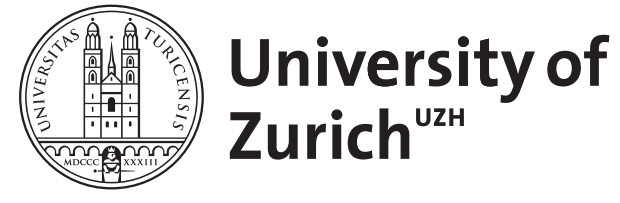

\title{
Das Schulkind mit ADHS
}

\author{
Walitza, Susanne ; Drechsler, Renate ; Ball, Juliane
}

\begin{abstract}
Die Aufmerksamkeitsdefizit- und Hyperaktivitätsstörung (ADHS) ist eine der häufigsten psychischen Störungen im Schulalter. Die Kernsymptome Hyperaktivität, Aufmerksamkeitsstörung und erhöhte Impulsivität treten vor dem 7. Lebensjahr auf und beeinträchtigen deutlich die Alltagsbewältigung (Schule, Familie, Freizeit) des Kindes. Bei $70 \%$ aller Betroffenen treten zudem mindestens eine weitere psychische Störung bzw. Lern- und Leistungsstörungen auf. Die ADHS ist in der Kinder- und Jugendpsychiatrie eines der am meisten beforschten Störungsbilder. In einer Vielzahl genetischer Studien und Bildgebungsstudien (MRT) zeigen Unterschiede zwischen Kindern mit ADHS und gesunden Kindern deutlich, dass ADHS eine neurobiologisch begründete Funktionsstörung ist. Nichtsdestotrotz spielen Umweltbedingungen wie familiäre und schulische Faktoren eine bedeutende Rolle bei der Aufrechterhaltung und Verstärkung der Symptomatik. Die Behandlung erfolgt multimodal, kindzentriert, unter Einbezug der Eltern und wenn möglich der Lehrpersonen. Die Gesellschaft mit ihren spezifischen Anforderungen, auch in der Schule hat sich nicht eingestellt auf Kinder, die anders sind, sehr bewegt und leicht ablenkbar. Diese Kinder haben jedoch auch Ressourcen. Sind sie einmal von einer Sache fasziniert, können sie in diesem Bereich durchaus Durchhaltevermögen und hohe Leistungsbereitschaft zeigen.
\end{abstract}

DOI: https://doi.org/10.1024/0040-5930/a000316

Other titles: The school child with ADHD

Posted at the Zurich Open Repository and Archive, University of Zurich

ZORA URL: https://doi.org/10.5167/uzh-74507

Journal Article

Accepted Version

Originally published at:

Walitza, Susanne; Drechsler, Renate; Ball, Juliane (2012). Das Schulkind mit ADHS. Therapeutische Umschau. Revue thérapeutique, 69(8):467-473.

DOI: https://doi.org/10.1024/0040-5930/a000316 
Prof. Dr. S. Walitza, Kinder- und Jugendpsychiatrischer Dienst Kanton Zürich, Neumünsterallee 9, 8032 Zürich, susanne.walitza@kjpdzh.ch, Telefon 0434992730 , Fax 0434992602

\section{Das Schulkind mit ADHS}

Susanne Walitza, Renate Drechsler und Juliane Ball

Kinder- und Jugendpsychiatrischer Dienst Kanton Zürich, Universitätsklinik Zürich

Zusammenfassung: Die Aufmerksamkeitsdefizit- und Hyperaktivitätsstörung (ADHS) ist eine der häufigsten psychischen Störungen im Schulkindalter. Die Kernsymptome Hyperaktivität, Aufmerksamkeitsstörung und erhöhte Impulsivität treten vor dem 7. Lebensjahr auf und beeinträchtigen deutlich die Alltagsbewältigung (Schule, Familie, Freizeit) des Kindes. Bei 70 $\%$ aller Betroffenen treten zudem mindestens eine weitere psychische Störung bzw. Lernund Leistungsstörungen auf. Die ADHS ist in der Kinder- und Jugendpsychiatrie eines der am meisten beforschten Störungsbilder.

Unterschiede zwischen Kindern mit ADHS und gesunden Kindern in einer Vielzahl genetischer und Bildgebungsstudien (MRT) zeigen deutlich, dass ADHS eine neurobiologisch begründete Funktionsstörung ist. Nichtsdestotrotz spielen Umweltbedingungen, familiäre und schulische Faktoren eine bedeutende Rolle bei der Aufrechterhaltung und Verstärkung der Symptomatik. Die Behandlung erfolgt multimodal, kindzentriert, unter Einbezug der Eltern und wenn möglich der Lehrpersonen. Die Gesellschaft mit ihren spezifischen Anforderungen, auch in der Schule an das Schulkind, hat sich nicht eingestellt auf Kinder, die anders sind, sehr bewegt und leicht ablenkbar. Diese Kinder haben jedoch auch Ressourcen. Sind sie einmal von einer Sache fasziniert, können sie in diesem Bereich durchaus Durchhaltevermögen und hohe Leistungsbereitschaft zeigen. 
Abstract

\section{The school child with ADHD}

Attention Deficit / Hyperactivity Disorder (ADHD) is one of the most frequent psychiatric disorders at school age. The core symptoms of hyperactivity, impulsivity and inattention emerge before the age of seven and are associated with severe impairment of the child's everyday functioning (family, school, leisure time). Seventy percent of the affected children show at least one comorbid psychiatric condition or learning disorder / academic impairment. ADHD is one of the most widely investigated disorders in child and adolescent psychiatry.

A multitude of imaging ( $\mathrm{fMRI}$ ) and genetic studies comparing children with ADHD and typically developing children have provided clear evidence for the neurobiological foundation of ADHD. Nevertheless, environmental, familial and academic factors play an important role with regard to the persistence and severity of the disorder. Treatment should be preferentially multimodal, with interventions targeting the child, the parents, and, if possible, the teacher.

Society with its specific requirements and constraints, and also school, are not well adjusted for children that are different, hyperactive and easily distracted. However, many of these children also have resources. When fascinated by a task, they may show persistence and commitment. 


\section{Fallbeispiel}

Der 9-jährige Jan wurde von seinen Eltern in der Poliklinik des KJPD Zürich vorgestellt. Die Eltern beschrieben ihren Sohn als interessiertes, dynamisches, motorisch äusserst aktives und impulsives Kind, welches Schwierigkeiten habe, sich allein zu beschäftigen und aber zum Beispiel gern mit Mittelpunkt stehe. Er habe wenige Freunde, andere Kinder würden inn ablehnen, weil er sehr dominant und bestimmend in Spielaktivitäten sei und zu laut und impulsiv auftrete. Er zeige zu Hause und in der Schule Konzentrationsprobleme, könne nur unzureichend an einer Aufgabe bleiben. Zumeist beginne er mehrere Sachen gleichzeitig, bringe sie aber nicht zu Ende. Auch in den täglichen Routinen wie z. B. Anziehen, Zähne putzen, Schultasche parat machen, zeichne er sich durch eine leichte Ablenkbarkeit aus, so dass einfachste Handlungsabläufe zeitaufwendig werden und Konfliktpotential darstellen. In der Schule falle auf, dass Jan trotz guter Begabung nur unzureichende Leistungen erbringe. Er mache viele Flüchtigkeitsfehler, vergesse Schulmaterialien, stehe im Unterricht auf und störe andere Kinder. Die Umsetzung der Anweisungen der Lehrerin, aber auch das Erledigen der Hausaufgaben, mache inm viel Mühe. Am Abend habe er Schwierigkeiten ins Bett zu gehen. Er wolle lieber aufbleiben, habe Angst, er könne etwas verpassen.

\section{Einleitung}

Zu den bekanntesten kinder- und jugendpsychiatrischen Störungen zählt zweifelsohne die Aufmerksamkeitsdefizit- und Hyperaktivitätsstörung (ADHS), die gekennzeichnet ist durch die Kernsymptomatik in den Bereichen Hyperaktivität, Impulsivität und Unaufmerksamkeit. Eine erste kinder- und jugendpsychiatrische Vorstellung und Abklärung erfolgt zumeist nach Eintritt in die Schule, wenngleich sich für die Diagnosestellung die Symptomatik bereits vor dem 7. Lebensjahr manifestiert haben muss und es in mehreren Lebenssituationen zu Beeinträchtigungen bei dem betroffenen Kind selbst und / oder seinem sozialen Umfeld kommen muss. Die Kernsymptome der ADHS sind dimensional zu sehen und zeigen sich in unterschiedlichster Ausprägung. Entscheidend für die Diagnose ist die Beeinträchtigung der Alltagsbewältigung und das Leiden, das durch die Kernsymptome /Hyperaktivität, Aufmerksamkeitsdefizit und Impulsivität verursacht wird. Der Eintritt in die Schule stellt einen Entwicklungsübergang dar, mit neuen Entwicklungsaufgaben wie z.B. der Erwerb von Kulturtechniken im Lesen, Schreiben und Rechnen. Der Schulbesuch bedingt aber auch neue Anforderungen in den Bereichen Aufmerksamkeit und Konzentration sowie Verhaltensanpassung und -kontrolle an externe Anforderungen in einem zumeist hochstrukturierten Rahmen. In der Schule fallen die Kinder mit ADHS häufig durch erheblich störende Verhaltensweisen im Unterricht auf. Sekundär können Lern- und Leistungsschwierigkeiten entstehen, die wiederum mit schlechteren Schulleistungen 
einhergehen und so zur Entwicklung von weiteren Störungen in der emotionalen oder sozialen Entwicklung beitragen können [1].

Bis zu $50 \%$ der Kinder mit ADHS brauchen Nachhilfeunterricht, etwa $30 \%$ von innen müssen eine Klasse wiederholen, 30 - $40 \%$ besuchen spezielle Förder- und Erziehungsprogramme und $46 \%$ wurden schon mindestens einmal vom Unterricht suspendiert [2]

Sowohl in der Kinder- und Jugendpsychiatrie als auch zunehmend in kinderärztlichen Praxen, in Erziehungsberatungsstellen und bei den schulpsychologischen Diensten stellen Kinder mit ADHS die grösste Inanspruchnahmepopulation dar [3]. Die Prävalenz für das Kindes- und Jugendalter wird innerhalb von Europa bei $5 \%$ bis $7 \%$ angenommen [4]. Dabei sind Jungen deutlich (in klinischen Studien vier- bis neunmal) häufiger als Mädchen betroffen. Bei Mädchen wird die Störung in der Regel jedoch erst später diagnostiziert [5]. Die geschätzte Prävalenz im Erwachsenenalter liegt bei 1-2.5 \% [6].

\section{Diagnostik und Differentialdiagnostik}

Die beiden gängigen Klassifikationssysteme unterscheiden sich in den diagnostischen Einordnungen und in der Differenziertheit der Erfassung der Symptomatik und ihrer Subtypen. Während das ICD-10 das klinische Vollbild eines ADHS mit allen drei Kardinalsymptomen unter der Diagnose F90.0 „einfache Aktivitäts- und Aufmerksamkeitsstörung“ zusammenfasst und zusätzlich die Erfassung einer „Hyperaktivitätsstörungen mit Störung des Sozialverhaltens“ F90.1 zulässt, unterscheidet das DSM-IV zwischen dem „kombinierten Typus ADHS“ (314.01) (welcher der ADHS nach ICD-10 entspricht), dem „vorwiegend unaufmerksamen Typus“ (314.00) und dem „vorwiegend hyperaktiven / impulsiven Typus“ (314.01). Im ICD-10 unzureichend repräsentiert ist der vorwiegend unaufmerksame Typus (das sogenannte Träumerle), also das Kind mit vorwiegender Aufmerksamkeitsstörung, welches lediglich in der Verwendung der Restkategorie F98.8 „sonstige näher bezeichnete Verhaltens- und emotionale Störungen mit Beginn in der Kindheit und Jugend" zu finden ist. Auch dem Praktiker werden all diese Bezeichnungen, Abkürzungen und Subtypen schon begegnet sein. Hilfreich erscheint der DSM-IV jedoch aktuell, da damit eine individuellere Beschreibung der ADHS Symptomatik möglich ist, wohingegen mit dem ICD-10 eine ADHS beschrieben ist, die häufiger behandlungsbedürftig ist. Entsprechend den Leitlinien der Deutschen Gesellschaft für Kinder- und Jugendpsychiatrie, Psychosomatik und Psychotherapie e.V. [7] ist eine sorgfältige und umfangreiche Diagnostik unerlässlich. Dabei müssen neben der kinder- und jugendpsychiatrischen und störungsspezifischen Diagnostik, umschriebene Entwicklungsstörungen (Teilleistungsstörungen), eine Unter- oder Überforderung (mittels Leistungsdiagnostik) und somatische Ursachen ausgeschlossen werden. Daneben ist eine 
genaue Diagnostik der psychosozialen Bedingungen notwendig [8]. Es sollten immer mehrere Informationsquellen einbezogen werden, neben den Kindern und Eltern selbst kann auch die Schule einen wertvollen Beitrag zur Diagnostik leisten. Bei der Anamneseerhebung ist ein strukturiertes klinisches Interview, welches mit Eltern und / oder Kind zur Erfassung der Symptomatik und der komorbiden Störungen durchgeführt wird, nützlich sein. Weitere Informationsquellen stellen Selbst- und Fremdbeurteilungsbögen, Gespräche und Unterrichtsbeobachtungen dar. Neuropsychologische Verfahren zur Diagnostik des ADHS, beispielsweise die computerisierten Testbatterien KITAP [9] oder die TAP [10], geben zwar wichtige Hinweise auf das individuelle Leistungsprofil des Kindes, ihr Beitrag zu einer klassifikatorischen Diagnostik ist jedoch gering [11]. Dies hängt vor allem mit der Heterogenität neuropsychologischer Störungen bei ADHS zusammen, da nur ein Teil der Kinder in objektiven Testverfahren klinisch relevante Beeinträchtigungen aufweist [12]. Neben der individuellen Profilbeschreibung, die für die Therapieplanung bedeutsam sein kann, ist der Nutzen der Verfahren in einer systematischen Verhaltensbeobachtung und Verlaufsbeobachtung, nach Einleitung einer Therapie, zu sehen.

Ein ADHS tritt selten ohne komorbide Störungen auf, mindestens $70 \%$ bis $90 \%$ der Kinder weisen eine komorbide Störung auf [13]. In bis zu $50 \%$ der Fälle lässt sich neben dem ADHS eine oppositionelle Störung des Sozialverhaltens bzw. in 10-20\% eine stärker ausgeprägte Störung des Sozialverhaltens diagnostizieren. Aber auch internalisierende Störungen wie Angststörungen oder depressive Störungen sind mit $15 \%$ bis $30 \%$ komorbid häufig vorhanden [13]. Weiterführend lassen sich Ticstörungen (10-20\%), Störungen der Ausscheidungsfunktionen (bis zu $30 \%$ ), Sprech- und Sprachstörungen, motorische Defizite und die schon genannten Lern- und Leistungsstörungen, inklusive Teilleistungsstörungen (10-30 \%) deutlich gehäuft bei Kindern mit ADHS finden. Auch aufgrund der hohen Komorbiditäten stellt das unbehandelte ADHS ein Risikofaktor für die weitere allgemeine Entwicklung, selbstverständlich auch für die schulische Entwicklung dar. ADHS-typische Symptome können auch unspezifische Symptome oder Merkmale anderer psychiatrischer (z. B. Depressionen, Psychosen) oder nicht-psychiatrischer Störungsbilder (Epilepsie, Hirntumore, motorische Unruhe bei Neurodermitis) sein [14]. Im Rahmen der differentialdiagnostischen Erwägungen ist neben einer körperlich-neurologischen Untersuchung auch die Durchführung eines EEGs, die Erhebung eines Differentialblutbildes bzw. bei entsprechenden Hinweisen eine fachärztliche Hör- und Sehfähigkeitsprüfung vorzunehmen. So sind beispielsweise unspezifische Auffälligkeiten im EEG bei ADHSPatienten deutlich häufiger als in der Normalpopulation [15] gesehen worden. Ein EEG ist dennoch kein spezifisches Diagnoseinstrument für die ADHS. 
Bei Jan wurde der Hamburg Wechsler Intelligenztest für Kinder durchgeführt, in dem er mit 108 IQ-Punkten ein durchschnittliches Ergebnis erzielte. Eine individuelle Leistungsschwäche zeigt sich im Bereich Verarbeitungsgeschwindigkeit. In der Aufmerksamkeitsdiagnostik mit der KITAP werden vermehrt Auslassungen durch eine erhöhte Ablenkbarkeit sowie Fehlreaktionen durch impulsives Handeln deutlich. Durchgängig zeigt sich in der Verhaltensbeobachtung eine erhöhte motorische Unruhe. Im Rahmen der Emotionaldiagnostik zeigte er keine depressive Symptomatik, thematisierte aber in einem semiprojektiven Test verschiedene Ängste (vor Dunkelheit, Alleinsein) sowie damit verbundene Gefühle von Einsamkeit. Er beschrieb z.B. er würde gern Kind bleiben und nicht erwachsen werden. In der von den Eltern ausgefüllten "Child Behavior Checklist" [16, 17]zeigten sich vor allem externalisierende Verhaltensauffälligkeiten. In einem ADHSspezifischen Fragebogen für Eltern und Lehrer [18, 19] wurden überdurchschnittliche Werte auf den Skalen Unaufmerksamkeit $(T=80)$, Hyperaktivität / Impulsivität $(T=71)$, Exekutive Funktionen ( $T=65)$ und Aggressivität $(T=69)$ deutlich. Bei der Erfassung seiner exekutiven Funktionen zeigten sich sowohl im Eltern- als auch im Lehrerurteil Defizite in den Bereich „Inhibition“ (Fähigkeit zur Verhaltenshemmung), „Shift“ (Fähigkeit zum flexiblen Situationsoder Anforderungswechsel), „Initiieren“ (Fähigkeit zur selbstständigen Aufgabenbearbeitung und Lösungsgenerierung) und „Monitoring“ (Fähigkeit zur Fehlerüberprüfung und Korrektur sowie Wahrnehmung wie das eigene Verhalten bei anderen ankommt) deutlich.

\section{Ätiologie und Bedingungen für den Verlauf}

Die Ätiologie der ADHS ist nicht abschliessend geklärt. Sicher ist, dass es sich um eine multifaktorielle Ursachengenese handelt, die komplexe Gen x Umwelt-Interaktionen einschliesst [20]. Die hohe interkulturelle Prävalenz von bis zu $5 \%$ für das Vollbild der Störung im Schulalter spricht bereits für biologische Grundlagen der Erkrankung. Genetische, hirnelektrische und bildgebende Befunde verweisen ebenfalls auf zugrundeliegende biologische Ursachen. Die Ausprägung und Beeinträchtigung für das einzelne Kind ist wiederum stark von Umweltfaktoren wie Erziehung oder Anforderungen z.B. in der Schule beeinflusst [21]. Für ADHS und auch für die einzelnen Kernsymptome wurde eine sehr hohe Erblichkeit von zwischen 60 und $80 \%$ ermittelt [22]. Zusammenfassend ist die Rate bei den Verwandten der Betroffenen etwa um das Fünffache erhöht [22] . Dieser Aspekt ist nicht nur für die genetische Forschung relevant, sondern muss auch im therapeutischen Alltag eine Berücksichtigung finden. So sollte den selbstbetroffenen Eltern eine entsprechende Hilfestellung und Unterstützung gewährt werden. In der Bildgebung können Reifungsunterschiede in der grauen Substanz bei Kindern mit ADHS im Vergleich zu gesunden Kindern gesehen werden. Die Reifungsverzögerung ist vor allem in frontalen 
Bereichen ausgeprägter und beträgt bis zu 2 Jahren [23] . Der früher häufiger gehörte Satz von Klinikern „ADHS wächst sich aus“, hat also durchaus für eine Subgruppe von Patienten, nun auch wissenschaftlich belegt, eine Berechtigung. Genauso ist sicher davon auszugehen, dass bei einer grossen Anzahl von Patienten bei zwischen 40 bis $60 \%$ einzelne der Symptome auch im Erwachsenenalter bestehen bleiben. Elektrophysiologisch und neuropsychologisch kann man im Vergleich zu Gesunden Aufmerksamkeitsstörungen und Inhibitionseinschränkungen beobachten [24].

Hinsichtlich der Impulsivität und Hyperaktivität, aber auch bei Störungen im sozialen Verhalten spielen in der Erziehung vor allem die sogenannten Erziehungsfallen eine besondere Rolle. So fokussieren die Eltern vor allem auf das negative Verhalten, das reduziert werden soll. Das Kind befolgt die Regeln nicht, die Eltern reagieren oftmals darauf, wiederholen sich, die Verärgerung steigt, bis die Familie entweder streitet oder die Eltern aufgeben. Das Kind hat seinen Willen vermeintlich durchgesetzt und wird lernen, dass es nur beharren muss. Andererseits werden positive Verhaltensweisen dann (vielleicht auch aus Erschöpfung nach den Auseinandersetzungen) vergleichsweise weniger wahrgenommen und auch zu wenig gelobt. Das Kind lernt, dass sich dieses Verhalten nicht lohnt. Entsprechend können sich Erziehungsfallen auch im schulischen Kontext manifestieren. Die Lehrperson ist froh, wenn es keinen Ärger mit dem ADHS Kind gibt und wird erst wieder auf das Kind aufmerksam, wenn es stört oder nicht bei der Sache ist. Ständige schulische Misserfolgserlebnisse tragen dazu bei, dass das Kind andere Möglichkeiten sucht, um Aufmerksamkeit von Lehrern und Mitschülern zu erreichen. So sind diese Kinder oft die Klassenclowns, damit sie überhaupt eine Stellung in der Klasse beziehen, ausser das Träumerlein und der Rabaucke zu sein. Aufgrund der hohe Impulsivität und reduzierten Steuerungsfähigkeit lassen sie sich häufiger in Auseinandersetzungen verwickeln und auch früher als die Altersgleichen zu Nikotinkonsum und Austesten gefährlicher Situationen oder Mutproben verleiten.

Häufiger nehmen Kinder mit ADHS dann auch eine delinquente Entwicklung, wobei sie im Gegensatz zu Kindern mit reiner Störung des Sozialverhaltens, ihre Fehltritte in der Regel bereuen.

\section{Therapie}

Die Behandlung des ADHS wird in der Regel multimodal konzipiert, wobei verschiedene kind- und elternzentrierte Bestandteile als auch eine Beratung in Kindergarten und Schule beinhaltet sind. Die Auswahl der Behandlungsmodalitäten erfolgt in Abhängigkeit vom Ausmass der Kernsymptomatik, den vorliegenden begleitenden Störungsbilder und dem erlebten Leidensdruck durch Kinder, Eltern und sozialen Umfeld [25]. Darüber hinaus werden die Ressourcen im familiären Umfeld, Stärken der Eltern und der Erfolg bisheriger 
Interventionen in die Behandlungsplanung miteinbezogen. Zentraler Bestandteil jeglicher Intervention ist in einem ersten Schritt die Beratung und Psychoedukation. Nach Abschluss der Diagnostik und Stellung der Diagnose werden Kind und Eltern in einem gemeinsamen Gespräch über die Ergebnisse der Diagnostik und das Störungsbild informiert. Es soll auf Basis der Ergebnisse und in Zusammenschau mit den anamnestischen Daten ein individuelles Störungskonzept vermittelt werden, welches neben den Schwächen und Problemen des Kindes auch die Stärken des Kindes und die Ressourcen der Familie berücksichtigt. Auf dieser Grundlage können dann gemeinsam mit der Familie grundlegende Strategien zum Umgang mit dem Kind sowie weitere therapeutisch notwendige Massnahmen abgeleitet werden. Häufig sind Eltern und natürlich auch die Kinder mit der Fülle an Informationen überfordert und es lohnt sich, die einzelnen Aspekte auf mehrere Sitzungen zu verteilen bzw. Inhalte wiederholt zu vermitteln.

Je jünger das Kind ist, umso mehr wird sich die Behandlung auf Elterntrainings (Abbau von Erziehungsfallen, Aufbau von positiver Verstärkung und konsequenter, aber liebevoller Erziehung, Strukturierung der Hausaufgabensituation etc.) konzentrieren. Verhaltenspläne, die vor allem mit der Verstärkung positiven Verhaltens arbeiten, sollten altersadäquat aufgebaut sein. Die Kinder können sehr gut bei der Gestaltung beteiligt werden, das erhöht auch die Compliance. Die Pläne sollten erreichbare (weniger ist mehr) und gut verständliche, nicht zu komplizierte Ziele enthalten. Mit zunehmendem Alter kann das Kind dann Selbstmanagementverfahren erlernen.

Die Indikationsstellung für die Therapie orientiert sich neben dem Alter und der Entwicklung entsprechend der Leitlinien der deutschen Fachgesellschaft an der Symptomatik des Kindes (Tab.1), wobei initial immer eine Verhaltenstherapie empfohlen wird. Dies steht im Widerspruch zu den amerikanischen Leitlinien, die sich stärker an Wirksamkeitsstudien orientieren und auch die Versorgungsrealität berücksichtigen [7, 26]. Als allgemeiner Praxisgrundsatz kann formuliert werden, dass die Therapieplanung sich an der Problemstellung (wo lassen sich die stärksten Beeinträchtigungen finden und welche Massnahme kann dies verändern) orientieren sollte.

Tabelle 1: Behandlungsansätze und deren Indikation nach DGKJP, 2007 (Darstellung modifiziert nach [25]

\begin{tabular}{|l|l|}
\hline Behandlungsansatz & Indikation \\
\hline Medikamentöse Behandlung & Stark ausgeprägte Symptomatik mit \\
& krisenhafter Zuspitzung oder unzureichender \\
& Wirksamkeit psychosozialer Interventionen \\
& mit weiter bestehender beeinträchtigender \\
& Symptomatik \\
\hline
\end{tabular}




\begin{tabular}{|c|c|}
\hline $\begin{array}{l}\text { Selbstinstruktionstraining } \\
\text { Selbstaufmerksamkeitstraining } \\
\text { (bei Vorschulkinder Spieltraining) }\end{array}$ & $\begin{array}{l}\text { Hyperkinetische Auffälligkeiten auch bei } \\
\text { optimalen Arbeitsbedingungen beim } \\
\text { Therapeuten }\end{array}$ \\
\hline Schulische Interventionen & $\begin{array}{l}\text { Externalisierende Auffälligkeiten des Kindes } \\
\text { in der Schule }\end{array}$ \\
\hline Elterntraining & $\begin{array}{l}\text { Externalisierende Auffälligkeiten des Kindes } \\
\text { in der Familie }\end{array}$ \\
\hline $\begin{array}{l}\text { Störungsspezifische } \quad \text { Behandlung der } \\
\text { komorbiden Störung }\end{array}$ & Komorbiditäten \\
\hline Teilstationäre / stationäre Behandlung & $\begin{array}{l}\text { Unzureichende Therapieresponse, deutliche } \\
\text { Gefährdung der psychosozialen Integration }\end{array}$ \\
\hline
\end{tabular}

Im folgenden soll nur auch einige der verschiedenen Behandlungsmöglichkeiten vertieft eingegangen werden.

\section{Selbstinstruktions- und Aufmerksamkeitstrainings sowie Selbstmanagment mit Kindern}

Das bekanntestes Selbstinstruktions- und Aufmerksamkeitstraining, welches im deutschen Sprachraum eingesetzt wird und seine Wirksamkeit belegt hat, ist das Training von Lauth und Schlottke [1]. Dies zielt darauf ab, dass Kinder lernen ihre Aufmerksamkeit zu fokussieren und impulsive Denk- und Handlungsstrategien zu unterdrücken, indem ein handlungsleitendes Selbstgespräch bei der Aufgabenbearbeitung eingeführt wird. Auch im Therapieprogramm mit hyperaktiven und aufmerksamkeitsgestörten Kindern [27] werden die Handlungsschritte: 1) Was soll ich tun? - 2) Wie ist mein Plan? - 3) Sorgfältig Schritt für Schritt zum Ziel - 4) Stop, überprüfen! - 5) Bewertung Prima! mit Hilfe von Selbstinstruktionskarten konkret in Problemsituationen geübt. Zumeist werden in den Programmen Aufgaben zur Bearbeitung vorgegeben, die eine konzentrative Leistung erfordern, aber auch die Möglichkeit bieten, die erlernten Strategien einzusetzen. Trotz der hohen Beliebtheit der Selbstinstruktions- und Aufmerksamkeitstrainings ist die klinische Wirksamkeit noch nicht überzeugend nachgewiesen [28]. Aus eigener klinischer Erfahrung, schätzen die Kinder es allerdings sehr, wenn innen auch etwas zugetraut wird und sie ein Selbstmanagementverfahren erlernen.

\section{Neurofeedback}

Auf der Basis von Forschungsergebnissen, dass bei einer Vielzahl der Kinder mit Aufmerksamkeitsstörungen im Vergleich zu gesunden Kindern Auffälligkeiten im EEG zu finden sind (z.B. [29, 30]), stellt das Neurofeedback auf der Grundlage des EEGs einen weiteren Behandlungsansatz dar. Die im Training erfassten EEG-Signale, meist EEGFrequenzbänder oder langsame kortikale Potentiale, werden dem Kind auf einem 
Probandenbildschirm zurückgemeldet, beispielsweise als ein Auto, das je nach Hirnaktivität schnell oder langsam fährt oder einen Fussballer, der ein Tor schiesst, wenn der gewünschte Schwellenwert erreicht ist. Indem im Neurofeedbacktraining die Hirnaktivität wahrnehmbar und somit beeinflussbar gemacht wird, sollen die Kinder lernen, ihre Aufmerksamkeit zu steuern. Grundlage des Neurofeedbacks stellt das operante Konditionieren dar. Erwünschte EEG-Aktivität bei gleichzeitiger Unterdrückung unerwünschter Aktivität bzw. die Veränderung von EEG-Aktivität in eine gewünschte Richtung werden durch Darbietung verständlicher Rückmeldesignale und Bonuspunkte verstärkt. Aber auch eine bessere Selbstwahrnehmung und verbesserte Strategien der Selbstregulation tragen zur Wirkung bei (vgl. Überblick bei Drechsler, [31])

\section{Schule}

Nach einer Studie von [32]kennen $80 \%$ der Lehrpersonen die Symptome der ADHS, aber sie wissen wenig über die Ursachen oder Hilfestellungen im Unterricht. Je besser sie über die Symptom informiert waren, umso schlechter schätzten sie ihre pädagogischen Fähigkeiten ein. Exemplarisch empfehlen Lehrpersonen ihren Kollegen u.a. folgende Unterrichtsstrategien: Das Kind mit ADHS sollte eher vorne sitzen, in der Nähe zur Lehrperson, bzw. sollte die Nachbarschaft des Kindes berücksichtigt werden.

Mögliche Ablenkungen können reduziert werden, dazu gehört auch eine Unterstützung bei der Pultorganisation. Eine gute Sitzhaltung (Gelenke rechter Winkel, Bodenkontakt mit Füssen, Wirbelsäule Kontakt mit Rückenlehne) hilft die Aufmerksamkeit zu erhöhen und länger aufrecht zu erhalten. Neben einem klaren Tagesablauf, helfen klare Regeln im Klassenzimmer. Falls mit Wutausbrüchen zu rechnen ist, sollten Auszeiten“ festgelegt werden. Ein Gesichtsverlust des Patienten ist dabei auf jeden Fall zu vermeiden. Wenn dem Kind Verantwortungsbereiche übergeben werden, kann dies helfen ein positives Selbstbild zu entwickeln.

Bezüglich Kommunikation mit einem betroffenen Kind ist es hilfreich, kurze Distanzen zu haben und viel, aber eher kurzen Blickkontakt zu nutzen. Das Kind sollte wenn möglich immer mit dem Namen angesprochen werden. Insgesamt ist eine eher unkomplizierte Sprache bis hin zur "Ein-Satz-Regel“ sinnvoll. Wenn aufgrund einer besonderen Hyperaktivität eine grosser Bewegungsdrang vorliegt, können Bewegungsmöglichkeiten geschaffen werden und wenn nötig können auch 2 Plätze im Klassenzimmer vorgesehen werden. Argumente wie, das ist ja viel zu aufwändig für ein Kind oder führt das nicht zur Benachteiligung der anderen Kinder, sollte nachgegangen werden. Folgende Punkte können Lehrpersonen insgesamt entlasten und zu einer guten Zusammenarbeit mit den Eltern führen:

- Überprüfen der Zusammensetzung der Klasse. 
- Überprüfen der Organisation des Klassenzimmers.

- Gestaltung des Unterrichtes strukturiert und abwechslungsreich.

- Probleme sollten frühzeitig angesprochen werden.

- Kontakt zu den Eltern sollte regelmässig stattfinden.

\section{Medikamentöse Behandlung}

Eine Medikation darf nur nach einer umfassenden ADHS Abklärung eingeleitet werden. Ab dem Schulalter (6 Jahre) sind betäubungsmittelpflichtige Stimulanzien (Methylphenidate $(\mathrm{MPH})$ : Ritalin ${ }^{\circledR}$, Ritalin LA $^{\circledast}$, Medikinet ${ }^{\oplus}$, Concerta $\left.{ }^{\circledR}\right)$ und selektive NoradrenalinWiederaufnahmehemmer (Atomoxetine ${ }^{\circledast}$ (ATX)) im Rahmen eines mulimodalen Behandlungskonzeptes zugelassen. Sowohl Stimulanzien als auch Atomoxetine haben aufgrund der guten Studienlage einen Evidenzgrad I bezogen auf die Wirksamkeit der Behandlung von ADHS Kernsymptomen. Die Medikation wird schrittweise langsam eindosiert. Bei Erreichen der Enddosis mit MPH empfiehlt sich die Umstellung auf ein langwirksames retardiertes MPH, welche 8 (Ritalin $\mathrm{LA}^{\circledR}$, Medikinet ${ }^{\circledR}$ ) oder bis zu 12 Stunden $\left(\right.$ Concerta $\left.^{\circledR}\right)$ wirken. Mit MPH in Kombination mit Verhaltenstherapie können auch Symptome der häufigen komorbiden Störungen reduziert werden, die Medikationsdosis kann reduziert werden und die Wirkung ist auch längerfristig positiv. Atomoxetin (Strattera ${ }^{\circledR}$ ) wirkt 24 Stunden und damit vom Aufstehen durch die ganze Nacht. Im Gegensatz zu MPH, dessen Wirkung sofort einsetzt und nach Ausdosieren seine volle Wirksamkeit erreicht hat, muss das Kind ATX 8 bis 12 Wochen einnehmen bis die volle Wirkung auf die Kernsymptome erreicht wird. MPH ist in der Schweiz das Medikament der ersten Wahl, bei Nonrespons oder Unverträglichkeit soll auf ATX umgestellt werden. In anderen Ländern ist ATX bei Auftreten von komorbiden Angst- und Ticstörungen sowie bei Suchterkrankungen ebenfalls erste Wahl [33]. In der Schweiz ist ATX im Vergleich zu MPH auch bei ADHS mit komorbider schweren Depression zugelassen. Nach den European Guidelines zum Management von Nebenwirkungen bei ADHS Medikation [34] erfordert das Nebenwirkungsprofil der Medikationsgruppen in der Kurzzeitbehandlung (12 Monate) nur Kontrollen von RR und Puls sowie bei Stimulanzien von Gewicht und Wachstum. Bei ATX gibt es eine Subgruppe von Patienten, die mit deutlich erhöhter Herzfrequenz und erhöhtem Blutdruck reagiert. Herzund Kreislauferkrankungen sowie Leber und Nierenfunktionsstörungen müssen eruiert werden. Sollten solche bei Patient oder auch Familienangehörigen vorliegen werden weitergehende diagnostische Prozesse nötig. 


\section{Literaturverzeichnis}

1. Lauth GW, Schlottke PF. Training mit aufmerksamkeitsgestörten Kindern. 5., vollst. überarb. Aufl. Weinheim: Beltz, 2002.

2. Barkley RA. Attention-deficit hyperactivity disorder. Sci Am 1998; 279(3): 66-71.

3. Döpfner M, Pluck J, Berner W, Fegert JM, Huss M, Lenz K, et al. Psychische Auffälligkeiten von Kindern und Jugendlichen in Deutschland - Ergebnisse einer repräsentativen Studie: Methodik, Alters-, Geschlechts- und Beurteilereffekte. Z Kinder Jugendpsychiatr Psychother 1997; 25(4): 218-33.

4. Polanczyk G, Rohde LA. Epidemiology of attention-deficit/hyperactivity disorder across the lifespan. Curr Opin Psychiatry 2007; 20(4): 386-92.

5. Döpfner M, Breuer D, Wille N, Erhart M, Ravens-Sieberer U. How often do children meet ICD-10/DSM-IV criteria of attention deficit-/hyperactivity disorder and hyperkinetic disorder? Parent-based prevalence rates in a national sample: results of the BELLA study. Eur Child Adolesc Psychiatry 2008; 17 Suppl 1: 59-70.

6. Simon V, Czobor P, Balint S, Meszaros A, Bitter I. Prevalence and correlates of adult attention-deficit hyperactivity disorder: meta-analysis. Br J Psychiatry 2009; 194(3): 204-11.

7. Deutsche Gesellschaft für Kinder- und Jugendpsychiatrie PuP. Leitlinien zu Diagnostik und Therapie von psychischen Störungen im Säuglings-, Kindes- und Jugendalter. 3., überarb. und erw. Aufl. Köln: Deutscher Ärzte-Verlag, 2007.

8. Remschmidt H. Multiaxiales Klassifikationsschema für psychische Störungen des Kindes- und Jugendalters nach ICD-10 der WHO mit einem synoptischen Vergleich von ICD10 mit DSM-IV. 5., vollst. überarb. und erw. Aufl. Bern: Huber, 2006.

9. Zimmermann P, Gondan M, Fimm B. Testbatterie zur Aufmerksamkeitsprüfung für Kinder (KiTAP). Herzogenrath: Psytest, 2002.

10. Zimmermann P, Fimm B. Testbatterie zur Aufmerksamkeitsprüfung (TAP), Version 2.2. Herzogenrath: Psytest, 2009.

11. Drechsler R, Rizzo, P, Steinhausen, HC. Zur klinischen Validität einer computergestützten Aufmerksamkeitstestbatterie für Kinder (KITAP) bei 7 bis 10jährigen Kindern mit Aufmerksamkeitsdefizit-/Hyperaktivitätsstörung (ADHS). Kindheit und Entwicklung 2009; 18: 153-61.

12. Willcutt EG, Doyle AE, Nigg JT, Faraone SV, Pennington BF. Validity of the executive function theory of attention-deficit/hyperactivity disorder: a meta-analytic review. Biol Psychiatry 2005; 57(11): 1336-46.

13. Taurines R, Schmitt J, Renner T, Conner AC, Warnke A, Romanos M. Developmental comorbidity in attention-deficit/hyperactivity disorder. Atten Defic Hyperact Disord 2010; 2(4): 267-89. 
14. Romanos M, Schwenck C, Walitza S. Diagnostik der Aufmerksamkeitsdefizit/Hyperaktivitätsstörung im Kindes- und Jugendalter. Nervenarzt 2008; 79(7): 782-90.

15. Holtmann M, Matei A, Hellmann U, Becker K, Poustka F, Schmidt MH. Rolandic spikes increase impulsivity in ADHD - a neuropsychological pilot study. Brain Dev 2006; 28(10): 633-40.

16. Achenbach TM. Manual for the Child Behavior Checklist/4-18 and 1991 Profile. Burlington: University of Vermont, Department of Psychiatry, 1991.

17. Arbeitsgruppe Deutsche Child Behavior Checklist. Elternfragebogen über das Verhalten von Kindern und Jugendlichen, deutsche Bearbeitung der Child Behavior Checklist (CBCL/4-18). Einführung und Anleitung zur Handauswertung. 2. Auflage mit deutschen Normen, bearbeitet von Döpfner M, Plück J, Bölte S, Lenz K, Melchers P und Heim K. Köln: Arbeitsgruppe Kinder-, Jugend- und Familiendiagnostik (KJFD), 1998.

18. Conners CK. Conners 3rd Edition. North Tonawanda: Multi-Health Systems, 2008.

19. Lidzba K, Christiansen H, Drechsler R. Conners 3D. Bern: Huber, im Druck.

20. Renner TJ, Gerlach M, Romanos M, Herrmann M, Reif A, Fallgatter AJ, et al. Neurobiologie des Aufmerksamkeitsdefizit-/Hyperaktivitatssyndroms. Nervenarzt 2008; 79(7): 771-81.

21. Faraone SV, Perlis RH, Doyle AE, Smoller JW, Goralnick JJ, Holmgren MA, et al. Molecular genetics of attention-deficit/hyperactivity disorder. Biol Psychiatry 2005; 57(11): 1313-23.

22. Biederman J, Faraone SV. Attention-deficit hyperactivity disorder. Lancet 2005; 366(9481): 237-48.

23. Shaw P, Eckstrand K, Sharp W, Blumenthal J, Lerch JP, Greenstein D, et al. Attention-deficit/hyperactivity disorder is characterized by a delay in cortical maturation. Proc Natl Acad Sci U S A 2007; 104(49): 19649-54.

24. Doehnert M, Brandeis D, Imhof K, Drechsler R, Steinhausen HC. Mapping attentiondeficit/hyperactivity disorder from childhood to adolescence: no neurophysiologic evidence for a developmental lag of attention but some for inhibition. Biol Psychiatry 2009; 67(7): 60816.

25. Jans T, Kreiker S, Warnke A. Multimodale Therapie der Aufmerksamkeitsdefizit/Hyperaktivitatsstörung im Kindesalter. Nervenarzt 2008; 79(7): 791-800.

26. Pliszka S, AACAP Work Group on Quality Issues. Practice parameter for the assessment and treatment of children and adolescents with attention-deficit/hyperactivity disorder. J Am Acad Child Adolesc Psychiatry 2007; 46(7): 894-921.

27. Döpfner $M$, Schürmann $S$, Frölich J. Therapieprogramm für Kinder mit hyperkinetischem und oppositionellem Problemverhalten THOP. 4., vollst. überarb. Aufl. Weinheim: Beltz, 2007. 
28. Pelham WE, Jr., Fabiano GA. Evidence-based psychosocial treatments for attentiondeficit/hyperactivity disorder. J Clin Child Adolesc Psychol 2008; 37(1): 184-214.

29. Barry RJ, Clarke AR, Johnstone SJ. A review of electrophysiology in attentiondeficit/hyperactivity disorder: I. Qualitative and quantitative electroencephalography. Clin Neurophysiol 2003; 114(2): 171-83.

30. Barry RJ, Johnstone SJ, Clarke AR. A review of electrophysiology in attentiondeficit/hyperactivity disorder: II. Event-related potentials. Clin Neurophysiol 2003; 114(2): 184-98.

31. Drechsler R. Ist Neurofeedbacktraining eine wirksame Therapiemethode zur Behandlung von ADHS? Ein Überblick über aktuelle Befunde. Z Neuropsychol 2011; 22: 131-46.

32. Ohan JL, Visser TA, Strain MC, Allen L. Teachers' and education students' perceptions of and reactions to children with and without the diagnostic label "ADHD". J Sch Psychol 2011; 49(1): 81-105.

33. Walitza S, Romanos M, Warnke A. Aufmerksamkeits-Defiziz/HyperaktivitätsStörungen. In: Gerlach M, Mehler-Wex C, Walitza S, eds. Neuro-Psychopharmaka im Kindes- und Jugendalter Grundlagen und Therapie. 2. Aufl. ed. Wien: Springer, 2009: 36582.

34. Graham J, Banaschewski T, Buitelaar J, Coghill D, Danckaerts M, Dittmann RW, et al. European guidelines on managing adverse effects of medication for ADHD. Eur Child Adolesc Psychiatry 2011; 20(1): 17-37. 